\title{
A complement to the Grigoriev theorem for the Kabanov model.
}

\author{
Emmanuel LEPINETTE, ${ }^{1}$ Jun ZHAO $^{2}$ \\ ${ }^{1}$ Ceremade, UMR CNRS 7534, Paris Dauphine University, PSL National Research, \\ Place du Maréchal De Lattre De Tassigny, \\ 75775 Paris cedex 16, France, and \\ Gosaef, Tunis-El Manar University, 2092-ElManar, Tunisia \\ Email: emmanuel.lepinette@ceremade.dauphine.fr \\ 2 Department of Applied Mathematics, Nanjing University of Science and Technology, \\ Nanjing 210094, Jiangsu, P.R. China. \\ Email: zhaojun.njust@hotmail.com
}

The authors thank Y. Kabanov, C. Kühn and A. Molitor for helpful discussions.

\begin{abstract}
We provide an equivalent characterisation of absence of arbitrage opportunity for the bid and ask financial market model analog to the Dalang-Morton-Willinger theorem formulated for discrete-time financial market models without friction. This result completes and improves the Grigoriev theorem for conic models in the two dimensional case by showing that the set of all terminal liquidation values is closed.
\end{abstract}

Keywords and phrases: Proportional transaction costs, Absence of arbitrage opportunities, Liquidation value, Bid and ask prices, Consistent price systems.

2000 MSC: 60G44, G11-G13.

\section{Introduction}

The Dalang-Morton-Willinger theorem [5] asserts, for the discrete-time final horizon frictionless market models, that the no-arbitrage property (NA) is equivalent to the existence of an equivalent martingale measure and any of these properties ensures that the set of super-replicated claims $A_{T}$ is closed in probability.

The models with friction were first considered in the pioneering paper [10] and, later, were extensively studied, e.g. in the papers [12], [8], [16], [7], [14]. With proportional transaction costs, it is classical to express the portfolio processes as stochastic vectors of $\mathbb{R}^{d}, d \geq 1$. Indeed, in presence of transaction costs, the exchanges are allowed between the assets at different rates so that 
it is not possible to describe them directly through the liquidation values (see [13, Ch. 3]).

In the theory of markets with proportional transaction costs, the closest analog of NA is the property $\mathrm{NA}^{w}$, i.e. the absence of strong arbitrage opportunities usually denoted. With the latter concepts, the DMW theorem can be extended but only for two-asset models and only partially. The corresponding result is known as the Grigoriev theorem, claiming that NA ${ }^{w}$ holds iff there is a consistent price system, accompanied by unexpected examples where the set of all vector-valued terminal portfolio processes is not closed under $\mathrm{NA}^{w}$, see [13, Ex. 1, Sect. 3.2.4 ]. Closedness is only proved under a strong absence of arbitrage opportunity, i.e. a robust no-arbitrage property $\mathrm{NA}^{r}$, see [13, Lem. 3.2.8]. Actually, one can mention that closedness is the important property to characterize the super-hedging prices (see [2] and [4]).

Here, we prove that the set of all terminal liquidation values is closed under $\mathrm{NA}^{w}$ for the two dimensional conic models. We then deduce a dual characterization of the prices super hedging a contingent claim when they are only expressed in the first asset.

\section{Model and basic properties}

\section{Notations.}

We define $e_{1}:=(1,0) \in \mathbb{R}^{2}, \mathbb{R}_{+}^{2}$ is the set of all vectors in $\mathbb{R}^{2}$ having only non negative components.

For a random set $E, L^{p}(E, \mathcal{F}), p \in[1, \infty)$ (resp. $p=\infty$ or $p=0$ ), is the normed space of all measurable selections of the random set $E$ and the superscript $p$ denotes the selections belonging to the corresponding Lebesgue space $L^{p}(E, \mathcal{F})$.

The Kabanov model. Let $\left(\Omega, \mathbb{F}:=\left(\mathcal{F}_{t}\right)_{t \leq T}, \mathrm{P}\right)$ be a discrete-time complete stochastic basis. We consider a risk-free asset $S^{0}=1$ and a risky asset defined by bid and ask adapted prices $S^{b}>0$ and $S^{a}>0$. This is the two-dimensional Kabanov model, [13, Ch. 3], equivalently defined by a $\mathbb{F}$ adapted set-valued process with values in the set of closed sectors (convex cones) $\left(\mathrm{G}_{t}\right)_{t \leq T}$ of the real plane $\mathbb{R}^{2}$ which are measurable in the sense that:

$$
\text { Graph } \mathrm{G}_{t}:=\left\{(\omega, x) \in \Omega \times \mathbb{R}^{2}: x \in \mathrm{G}_{t}(\omega)\right\} \in \mathcal{F}_{t} \otimes \mathcal{B}\left(\mathbb{R}^{2}\right), \quad t \leq T .
$$

In finance, $\mathrm{G}_{t}$ is interpreted as the set of all positions $x \in \mathbb{R}^{2}$ it is possible to liquidate at time $t$ without any debt. We have $\mathrm{G}_{t}=\left\{x \in \mathbb{R}^{2}: \mathrm{L}_{t}(x) \geq 0\right\}$ 
where the liquidation value process introduced in [1] is

$$
\mathrm{L}_{t}(x):=x^{1}+\left(x^{2}\right)^{+} S_{t}^{b}-\left(x^{2}\right)^{-} S_{t}^{a}, \quad x=\left(x^{1}, x^{2}\right) \in \mathbb{R}^{2} .
$$

It is easy to check that the liquidation value satisfies the following:

Lemma 2.1. $\quad$ Let $\mathrm{L}_{t}$ be defined by (2.1).

1. The mapping $x \mapsto \mathrm{L}_{t}(x)$ is concave hence continuous.

2. $\mathrm{L}_{t}\left(x^{0}, z\right)=x^{0}+\mathrm{L}_{t}((0, z))$ for all $x^{0}, z \in \mathbb{R}$.

3. $x-\mathrm{L}_{t}(x) e_{1} \in \partial \mathrm{G}_{t}$ for all $x \in \mathbb{R}^{2}$.

The boundary $\partial \mathrm{G}_{t}=\left\{x \in \mathbb{R}^{2}: \mathrm{L}_{t}(x)=0\right\}$ is composed of two rays $\mathbb{R}_{+}\left(S_{t}^{a},-1\right)$ and $\mathbb{R}_{+}\left(-S_{t}^{b}, 1\right)$. The dual $\mathrm{G}_{t}^{*}:=\left\{z \in \mathbb{R}^{2}: z x \geq 0, \forall x \in \mathrm{G}_{t}\right\}$ has the rays $\mathbb{R}_{+}\left(1, S_{t}^{b}\right)$ and $\mathbb{R}_{+}\left(1, S_{t}^{a}\right)$ as boundaries. We have $\mathrm{G}_{t}^{*} \backslash\{0\} \subseteq \operatorname{int} \mathbb{R}_{+}^{2}$ and $\mathrm{G}_{t}^{*}=\operatorname{cone}\left(\{1\} \times\left[S_{t}^{b}, S_{t}^{a}\right]\right)^{1}$.

Definition 2.2. An adapted self-financing portfolio process $\left(V_{t}\right)_{t \leq T}$ starts from an initial endowment $V_{0-}=V_{-1}$ and satisfies $\Delta V_{t} \in-\mathrm{G}_{t}, \forall t \leq T$ a.s.

We introduce the set of all terminal values at time $t \leq T$ of the portfolio processes starting from the zero initial endowment at time $u \leq t$, i.e.

$$
\mathcal{A}_{u}^{t}:=\sum_{s=u}^{t} L^{0}\left(-\mathrm{G}_{s}, \mathcal{F}_{s}\right) .
$$

The corresponding set of terminal liquidation values is:

$$
\mathcal{L}_{u}^{t}:=\left\{\mathrm{L}_{t}(V): V \in \mathcal{A}_{u}^{t}\right\}
$$

We introduce a condition $\mathbf{E}$ satisfied by classical examples of markets:

Definition 2.3. Let $T \geq 2$. We say that condition $\mathbf{E}$ holds if the following implications hold for all $t \leq T-1$, for all $u \geq t+1$ and for all $F_{u} \in \mathcal{F}_{u}$ :

(i) If $S_{t}^{a}=S_{u}^{b}$ on $F_{u}$, then there exists $r \geq u$ such that $S_{t}^{a} \geq S_{r}^{a}$ on $F_{u}$,

(ii) If $S_{t}^{b}=S_{u}^{a}$ on $F_{u}$, then there exists $r \geq u$ such that $S_{r}^{b} \geq S_{t}^{b}$ on $F_{u}$.

Remark 2.4. Note that $\left(S_{t}^{a},-1\right) \in \mathrm{G}_{r}$ iff $S_{t}^{a} \geq S_{r}^{a}$ and $\left(-S_{t}^{b}, 1\right) \in \mathrm{G}_{r}$ if and only if $S_{r}^{b} \geq S_{t}^{b}$.

\footnotetext{
${ }^{1}$ The notation cone $(A)$ designates the smallest cone containing $A$.
} 
Let us present some examples where condition $\mathbf{E}$ holds:

Example 1: This first example generalizes the model [8]. Let $\left(S_{t}\right)_{t \leq T}$ be a mid-price adapted process and consider a process $\left(\epsilon_{t}\right)_{t \leq T}$ of proportional transaction cost rates with values in $[0,1)$. We suppose that $\left(S_{t}\right)_{t \leq T}$ and $\left(\epsilon_{t}\right)_{t \leq T}$ are two independent processes and for every $t<u$, one of the random variables $S_{u} / S_{t}$ and $\left(1+\epsilon_{t}\right) /\left(1-\epsilon_{u}\right)$ does not admit any atom. The bid and ask prices are given by $S_{t}^{b}:=S_{t}\left(1-\epsilon_{t}\right), S_{t}^{a}:=S_{t}\left(1+\epsilon_{t}\right)$. Then, we show that $\mathrm{P}\left(S_{t}^{a}=S_{u}^{b}\right)=0$ if $u>t$ so that condition $\mathbf{E}$ trivially holds.

Example 2: We consider the Cox-Ross-Rubinstein model with bid-ask spreads of $\left[11\right.$, Sect. 4]. The bid and ask prices are $S_{t}^{b}=\left(1+\zeta_{t}^{b}\right) S_{t-1}^{a}, S_{t}^{a}=\left(1+\zeta_{t}^{a}\right) S_{t-1}^{b}$, where $\zeta=\left(\zeta^{b}, \zeta^{a}\right)$ is such that $0<S_{t}^{b} \leq S_{t}^{a}$ a.s. for all $t \leq T$. In [11, Sect. 4], $\zeta^{b}$ and $\zeta^{a}$ take two distinct values. Here, we suppose that $P\left(\zeta_{t}^{a}=0\right)=$ $P\left(\zeta_{t}^{b}=0\right)=0$ for all $t$ and, for all $u \geq 2,1+\zeta_{t+u}^{b} \geq \min _{r \geq t+u} S_{r}^{a} / S_{t+u-1}^{a}$ when $1+\zeta_{t+u}^{b}=S_{t}^{a} / S_{t+u-1}^{a}$ and $1+\zeta_{t+u}^{a} \leq \max _{r \geq t+u} S_{r}^{b} / S_{t+u-1}^{b}$ when we have $1+\zeta_{t+u}^{a}=S_{t}^{b} / S_{t+u-1}^{b}$. In that case, condition $\mathbf{E}$ holds. On the other hand, if we suppose that $P\left(\zeta_{t}^{a}=0\right)=P\left(\zeta_{t}^{b}=0\right)=0$ for all $t$ and $\zeta_{t}^{a}, \zeta_{t}^{a}$ are independent of $\mathcal{F}_{t-1}$, while the ratios $S_{t+r}^{a} / S_{t}^{a}$ and $S_{t+r}^{b} / S_{t}^{b}, r \geq 1$ admit densities, then condition $\mathbf{E}$ trivially holds.

Example 3: As in [8], we suppose that the bid and ask prices are $S_{t}^{b}=$ $S_{t}-\epsilon_{t}$ and $S_{t}^{a}=S_{t}+\epsilon_{t}, \quad t \leq T$. Here, $S$ and $\epsilon$ are two positive adapted processes such that $S^{b}>0$. Then, condition $\mathbf{E}$ trivially holds when $S$ and $\epsilon$ are independent and one of them does not admit any atom.

\section{Main result}

Definition 3.1. We say that the market model G satisfies the weak noarbitrage property $N A^{w}$ if $\mathcal{L}_{0}^{T} \cap L^{0}\left(\mathbb{R}_{+}, \mathcal{F}_{T}\right)=\{0\}$.

For the models with proportional transaction costs, $\mathrm{G}_{T}$ strictly dominates $\mathbb{R}_{+}^{2}$, i.e. $\mathbb{R}_{+}^{2} \backslash\{0\} \subset$ int $G_{T}$, and we may easily show the following:

Lemma 3.2. If $\mathrm{G}_{T}$ strictly dominates $\mathbb{R}_{+}^{2}$, then

$$
\mathcal{L}_{0}^{T} \cap L^{0}\left(\mathbb{R}_{+}, \mathcal{F}_{T}\right)=\{0\} \Longleftrightarrow \mathcal{A}_{0}^{T} \cap L^{0}\left(\mathbb{R}_{+}^{2}, \mathcal{F}_{T}\right)=\{0\} .
$$

Recall that a consistent price system (CPS) is a martingale $\left(Z_{t}\right)_{t \leq T}$ satisfying $Z_{t} \in \mathrm{G}_{t}^{*} \backslash\{0\}$ for all $t \leq T$. 
Theorem 3.3. ( Grigoriev theorem, [6], [13, Th. 3.2.15]) The following conditions are equivalent:

$1 N A^{w}$.

$2 \overline{\mathcal{A}_{0}^{T}} \cap L^{0}\left(\mathbb{R}_{+}^{d}, \mathcal{F}_{T}\right)=\{0\}^{2}$;

3 For any $\tilde{P} \sim \mathrm{P}$, there exists a bounded $C P S$ under $\tilde{P}$.

With friction, $\mathcal{A}_{0}^{T}$ is not necessarily closed, see [13, Ex. 1, Sect. 3.2.4].

Theorem 3.4. Suppose that condition $\mathbf{E}$ holds if $T \geq 2$. The following conditions are equivalent:

$1 N A^{w}$

$2 \mathcal{L}_{0}^{T}$ is closed in probability and $\mathcal{L}_{0}^{T} \cap L^{0}\left(\mathbb{R}_{+}, \mathcal{F}_{T}\right)=\{0\}$.

3 There exists $Q \sim \mathrm{P}$ with $d Q / d \mathrm{P} \in L^{\infty}$ such that $\mathrm{E}_{Q} \mathrm{~L}_{T}(V) \leq 0$ for all $\mathrm{L}_{T}(V) \in \mathcal{L}_{0}^{T} \cap L^{1}(\mathrm{P})$.

4 There exists $Q \sim \mathrm{P}$ with $d Q / d \mathrm{P} \in L^{\infty}$ such that, for all $t \leq T-1$, $\mathrm{E}_{Q}\left(S_{t+1}^{a} \mid \mathcal{F}_{t}\right) \geq S_{t}^{b}$ and $\mathrm{E}_{Q}\left(S_{t+1}^{b} \mid \mathcal{F}_{t}\right) \leq S_{t}^{a}$.

5 There exists $Q \sim \mathrm{P}$ with $d Q / d \mathrm{P} \in L^{\infty}$ and a $Q$-martingale $\tilde{S} \in\left[S^{b}, S^{a}\right]$.

In the following, we denote by $\mathcal{M}^{\infty}(P)$ the set of all $Q \sim \mathrm{P}$ such that $d Q / d \mathrm{P} \in L^{\infty}$ and $\mathrm{E}_{Q} \mathrm{~L}_{T}(V) \leq 0$ for all $\mathrm{L}_{T}(V) \in \mathcal{L}_{0}^{T}$. For any contingent claim $\xi \in L^{1}\left(\mathbb{R}, \mathcal{F}_{T}\right)$, we define the set $\Gamma_{\xi}$ of all initial endowments of portfolio processes whose terminal liquidation values coincide with $\xi$, i.e.

$$
\Gamma_{\xi}:=\left\{x \in \mathbb{R}: \exists V \in \mathcal{A}_{0}^{T}: \mathrm{L}_{T}\left(x e_{1}+V_{T}\right)=\xi\right\} .
$$

Corollary 3.5. Suppose that condition $\mathbf{E}$ holds. Let $\xi \in L^{0}\left(\mathbb{R}, \mathcal{F}_{T}\right)$ be such that $\mathrm{E}_{\mathrm{P}}|\xi|<\infty$. Then, under condition $N A^{w}, \Gamma_{\xi}=\left[\sup _{Q \in \mathcal{M}^{\infty}(P)} \mathrm{E}_{Q} \xi, \infty\right)$.

The following is suggested by C. Kühn and confirms the necessity of $\mathbf{E}$.

Example 3.6. There exists a financial market model satisfying $N A^{w}$ but condition $\mathbf{E}$ fails and such that $\mathcal{L}_{0}^{T}$ is not closed.

Proof. Let us define $\Omega=\left\{\omega_{k, i}: k \in \mathbb{N} \backslash\{0\}, i=1,2\right\}$ and $T=2$. Suppose that $\mathcal{F}_{0}=\{\emptyset, \Omega\}, \mathcal{F}_{1}=\sigma\left\{\left\{\omega_{k, 1}, \omega_{k, 2}\right\}: k \geq 1\right\}$ and $\mathcal{F}_{2}=2^{\Omega}$. The bid and ask prices are defined by

\footnotetext{
${ }^{2}$ The closure is taken in $L^{0}$.
} 


$$
\begin{aligned}
& S_{0}^{b}=S_{0}^{a}=1, \quad S_{1}^{b}=1, S_{1}^{a}=2, \\
& S_{2}^{b}\left(\omega_{k, i}\right)=S_{2}^{a}\left(\omega_{k, i}\right)=1+\frac{(-1)^{i+1}}{k}, \quad k \in \mathbb{N} \backslash\{0\}, i=1,2 .
\end{aligned}
$$

Moreover, we suppose that $P\left(\left\{\omega_{k, 1}\right\} \mid \mathcal{F}_{1}\right)=P\left(\left\{\omega_{k, 2}\right\} \mid \mathcal{F}_{1}\right)$ for all $k \geq 1$ so that $\mathrm{E}\left(S_{2}^{b} \mid \mathcal{F}_{1}\right)=1$. We deduce that $Z_{t}=\left(1, S_{t}^{b}\right)$ is a CPS so that $\mathrm{NA}^{w}$ holds by the Grigoriev theorem. This is an example where condition $\mathbf{E}$ does not hold at time $t=0$. Indeed, in the contrary case, as $S_{0}^{a}=S_{1}^{b}$ a.s., we should have a.s. the existence of $r \geq 1$ such that $S_{0}^{a} \geq S_{r}^{a}$. Necessary $r=2$ so that we should have $1 \geq S_{2}^{a}$ a.s., which is not the case.

Let us define $H^{1 n}=\left\{\omega_{k, 1}: k \leq n\right\}$ and $H^{2 n}=\left\{\omega_{k, 2}: k \leq n\right\}$ for all $n \in[1, \infty]$ and $H^{i}=H^{i \infty}, i=1,2$. We may show by contradiction that the payoff $H=1_{H^{1}}-1_{H^{2}}$ does not belong to $\mathcal{L}_{0}^{2}$. On the other hand, $H=\lim _{n} H^{n}$ where $H^{n}=1_{H^{1 n}}-1_{H^{2 n}}$. We claim that $H^{n} \in \mathcal{L}_{0}^{2}$. Indeed, it suffices to buy $n+1$ risky assets at time $t=0$, sell $n+1-k \geq 0$ assets at time $t=1$ on each $\left\{\omega_{k, 1}, \omega_{k, 2}\right\} \in \mathcal{F}_{1}$ such that $k \leq n$ and sell the $n+1$ assets otherwise. At last, liquidating the position at time $t=2$, we finally get the payoff

$$
\left[k\left(1+(-1)^{i+1} / k-k\right] 1_{k \leq n}=H^{n}\left(\omega_{k, i}\right) .\right.
$$

As $H=\lim _{n} H^{n}$, we conclude that $\mathcal{L}_{0}^{T}$ is not closed.

\section{Proofs of the main results}

\subsection{Proof of Theorem 3.4}

The implication $(2) \Rightarrow(3)$ follows from [13, Th. 2.1.4]. The implications $(3) \Rightarrow(1)$ and $(2) \Rightarrow(1)$ are trivial. The implication $(3) \Rightarrow(4)$ is deduced by considering the liquidation values at time $t+1$ of the positions $\left(S_{t}^{b},-1\right) 1_{F_{t}}$ and $\left(-S_{t}^{a}, 1\right) 1_{F_{t}}, F_{t} \in \mathcal{F}_{t}$. The implication $(4) \Rightarrow(5)$ is deduced from $[3$, Th. 4.5]. At last, if (5) holds, $Z=(\rho, \rho \tilde{S})$ is a CPS with $\rho_{t}=\mathrm{E}\left(d Q / d P \mid \mathcal{F}_{t}\right)$ so that $\mathrm{NA}^{w}$ holds. If $\mathrm{NA}^{w}$ holds, $\overline{\mathcal{L}_{0}^{T}} \cap L^{0}\left(\mathbb{R}_{+}, \mathcal{F}_{T}\right)=\{0\}$ by the Grigoriev theorem, where the closure is in $L^{1}$. We deduce (3) by [13, Th. 2.1.4].

Closedness. It remains to show that $(1) \Rightarrow(2)$, i.e. $\mathcal{L}_{0}^{T}$ is closed in probability. With one step, this is immediate as $\mathcal{L}_{T}^{T}=-L^{0}\left(\mathbb{R}_{+}, \mathcal{F}_{T}\right)$. We may show that, for any $\gamma \in \mathcal{L}_{0}^{T}, \gamma e_{1}=-g_{0}^{T} \in \mathcal{A}_{0}^{T}$ where $g_{u}^{t}, u \leq t$, is a general notation we 
introduce to designate a sum $g_{u}^{t}=\sum_{r=u}^{t} g_{r}$ with $g_{r} \in L^{0}\left(\mathrm{G}_{r}, \mathcal{F}_{r}\right), r \leq T$. Moreover, we may suppose w.l.o.g. that $g_{r} \in \partial \mathrm{G}_{t}$ for all $t \leq T-1$. By the Grigoriev theorem, there exists a CPS $Z$.

Two steps. Consider $\gamma_{T}^{\infty}=\lim _{n} \gamma_{T}^{n}$ where $\gamma_{T}^{n} e_{1}=-g_{T-1}^{T, n} \in \mathcal{L}_{T-1}^{T}$. Define the set $\Gamma_{T-1}:=\left\{\liminf \left|g_{T-1}^{n}\right|=\infty\right\} \in \mathcal{F}_{T-1}$. We normalize the sequences by setting $\tilde{\gamma}_{T}^{n}:=\gamma_{T}^{n} /\left|g_{T-1}^{n}\right|, \tilde{g}_{T-1}^{T, n}:=g_{T-1}^{T, n} /\left|g_{T-1}^{n}\right|$. As $\left|\tilde{g}_{T-1}^{n}\right|=1$, we may assume that $\tilde{g}_{T-1}^{n} \rightarrow \tilde{g}_{T-1}^{\infty} \in \mathrm{G}_{T-1}$, see [13, Lem. 2.1.2]. As $\lim _{n} \tilde{\gamma}_{T}^{n} e_{1}=0$, we deduce that $\tilde{g}_{T}^{n} \rightarrow \tilde{g}_{T}^{\infty} \in \mathrm{G}_{T}$ and $\tilde{g}_{T-1}^{\infty}+\tilde{g}_{T}^{\infty}=0$ where $\tilde{g}_{T-1}^{\infty} \in \partial \mathrm{G}_{T-1}$ and $\tilde{g}_{T}^{\infty} \in \mathrm{G}_{T}$. We set $\tilde{g}_{T-1}^{\infty}=\tilde{g}_{T}^{\infty}=0$ on $\Lambda_{T-1}=\Omega \backslash \Gamma_{T-1} \in \mathcal{F}_{T-1}$. Let $Z$ be a CPS. As $Z_{T}\left(\tilde{g}_{T-1}^{\infty}+\tilde{g}_{T}^{\infty}\right)=0$, we deduce that $Z_{T-1} \tilde{g}_{T-1}^{\infty}+\mathrm{E}\left(Z_{T} \tilde{g}_{T}^{\infty} \mid \mathcal{F}_{T-1}\right)=0$. By duality, we get that $Z_{T-1} \tilde{g}_{T-1}^{\infty}=Z_{T} \tilde{g}_{T}^{\infty}=0$. As $\tilde{g}_{T}^{\infty}=-\tilde{g}_{T-1}^{\infty}$ is $\mathcal{F}_{T-1^{-}}$ measurable, we get that $0=\mathrm{E}\left(Z_{T} \tilde{g}_{T}^{\infty} \mid \mathcal{F}_{T-1}\right)=Z_{T-1} \tilde{g}_{T}^{\infty}$. So, $Z_{T-1} \tilde{g}_{T}^{\infty}=Z_{T} \tilde{g}_{T}^{\infty}$ hence $Z_{T-1} \in\left(\mathbb{R}_{+} Z_{T}\right) \cap \mathrm{G}_{T}^{*}$. Therefore, $Z_{T-1} \gamma_{T}^{n} e_{1}=-Z_{T-1} g_{T-1}^{n}-Z_{T-1} g_{T}^{n} \leq 0$ by duality. Since $Z_{T-1} e_{1}>0$, we deduce that $\gamma_{T}^{n} \leq 0$. So, $\gamma_{T}^{n} e_{1}=-\hat{g}_{T-1}^{T, n}$ a.s., where $\hat{g}_{T-1}^{n}=g_{T-1}^{n} 1_{\Lambda_{T-1}} \in \partial \mathrm{G}_{T-1}$ and $\hat{g}_{T}^{n}=g_{T}^{n} 1_{\Lambda_{T-1}}+\left(-\gamma_{T}^{n} e_{1}\right) 1_{\Gamma_{T-1}}$ belongs to $L^{0}\left(\mathrm{G}_{T}, \mathcal{F}_{T}\right)$. By construction, $\lim \inf _{n}\left|\hat{g}_{T-1}^{n}\right|<\infty$ hence we may suppose that $\hat{g}_{T-1}^{n} \rightarrow \hat{g}_{T-1}^{\infty} \in L^{0}\left(\mathrm{G}_{T-1}, \mathcal{F}_{T-1}\right)$ by [13, Lem. 2.1.2]. We deduce that $\hat{g}_{T}^{n} \rightarrow \hat{g}_{T}^{\infty} \in L^{0}\left(\mathrm{G}_{T}, \mathcal{F}_{T}\right)$ hence $\gamma_{T}^{\infty}=-\hat{g}_{T-1}^{T, \infty} \in \mathcal{L}_{T-1}^{T}$.

General case. Condition $\mathbf{E}$ is only used for 3 steps and more and we argue by induction. Suppose closedness holds between the dates $t+1$ and $T \geq 2$ and let us show the closedness holds between $t$ and $T$. To do so, we suppose that $\lim _{n} \delta_{T}^{n}=\delta_{T} \in L^{0}\left(\mathbb{R} e_{1}, \mathcal{F}_{T}\right)$ where $\delta_{T}^{n}=-g_{t}^{T, n} \in \mathcal{A}_{t}^{T}$. We claim that $\delta_{T}^{n}=-\hat{g}_{t}^{T, n}+\epsilon_{T}^{n}$, where $-\hat{g}_{t}^{T, n} \in \mathcal{A}_{t}^{T}$ satisfies $\sup _{u \leq T} \liminf \inf _{n}\left|\hat{g}_{u}^{n}\right|<\infty$ a.s. and $\lim _{n} \epsilon_{T}^{n}=0$ a.s. Moreover, $\hat{g}_{t}^{n}=g_{t}^{n} 1_{\left\{\liminf _{n}\left|g_{t}^{n}\right|<\infty\right\}}$. This holds for $t=T-1$ as shown above.

(i) We first work on $\Lambda_{t}:=\left\{\liminf _{n}\left|g_{t}^{n}\right|<\infty\right\} \in \mathcal{F}_{t}$. Consider the smallest $u \geq t+1$ such that $P\left(\liminf _{n}\left|g_{u}^{n}\right|=+\infty \mid \Lambda_{t}\right)>0$. As $\liminf _{n}\left|g_{r}^{n}\right|<\infty$ a.s., we suppose that $g_{r}^{n} \rightarrow g_{r} \in L^{0}\left(\partial \mathrm{G}_{r}, \mathcal{F}_{r}\right)$ by [13, Lem. 2.1.2] if $r \leq u-1$. Then, we replace $g_{r}^{n}$ by $g_{r}$ if $r \leq u-1$, letting aside a residual error $\epsilon_{T}^{n} \rightarrow 0$ a.s. and we only need to consider the case $u \leq T-1$. We split $\Lambda_{t}$ into $\Lambda_{u} \in \mathcal{F}_{u}$ and $\Gamma_{u}=\Omega \backslash \Lambda_{u}$.

On $\Gamma_{u}$, we mormalize by dividing by $\left|g_{u}^{n}\right|$ and we get that $\tilde{\delta}_{T}^{n}=-\tilde{g}_{t}^{T, n}$ where $\tilde{\delta}_{T}^{n}=\delta_{T}^{n} /\left|g_{u}^{n}\right|$ and $\tilde{g}_{r}^{n}:=g_{r}^{n} /\left|g_{u}^{n}\right|$ for $r \leq T$. As $\tilde{\delta}_{T}^{n}$ and $\tilde{g}_{r}^{n}, r \leq u-1$, tend to 0 , we may suppose that $\lim \inf _{n}\left|\tilde{g}_{r}^{n}\right|<\infty$ by the induction hypothesis on $\Gamma_{u}$ if $r \geq u$. By [13, Lem. 2.1.2], we may suppose that $\tilde{g}_{r}^{n} \rightarrow \tilde{g}_{r}^{\infty} \in L^{0}\left(\mathrm{G}_{r}, \mathcal{F}_{r}\right)$ 
if $r \geq u$. Finally, we get that $\tilde{g}_{u}^{T, \infty}=0$ and $\tilde{g}_{u}^{\infty} \in L^{0}\left(\partial \mathrm{G}_{u}, \mathcal{F}_{u}\right)$. We get that $\tilde{g}_{T}^{\infty} \in L^{0}\left(\partial \mathrm{G}_{T}, \mathcal{F}_{T}\right)$ under $\mathrm{NA}^{w}$. Let us consider the stopping time $\tau$ as the first instant $\tau \geq u+1$ such that $\tilde{g}_{u}^{\tau, \infty}=0$. By Lemmas 4.1, 4.2, for all $r \geq u$, there exists $k_{r} \in L^{0}\left(\mathbb{R}, \mathcal{F}_{r}\right)$ such that $\tilde{g}_{r}^{\infty} 1_{r \leq \tau}=k_{r} \tilde{g}_{u}^{\infty} 1_{r \leq \tau}$. Consider the first instant $\sigma \in[u+1, \tau]$ such that $k_{\sigma}<0$. It exists since $k_{u}=1$ and $\tilde{g}_{u}^{\tau, \infty}=0$. We consider the case where $\tilde{g}_{u}^{\infty(1)}>0$ and $\tilde{g}_{u}^{\infty(2)}<0$, then $\tilde{g}_{\sigma}^{\infty(1)}<0$ and $\tilde{g}_{\sigma}^{\infty(2)}>0$. The symmetric case may be solved similarly.

Note that $\tilde{g}_{\sigma}^{\infty}=k_{\sigma} \tilde{g}_{u}^{\infty}$ hence $-k_{\sigma}^{-1} \tilde{g}_{\sigma}^{\infty}=-\tilde{g}_{u}^{\infty}$. By $\mathrm{NA}^{w}$, we get that $\mathrm{L}_{\sigma}\left(\tilde{g}_{\sigma}^{\infty}\right)=0$. Since $\mathrm{L}_{u}\left(\tilde{g}_{u}^{\infty}\right)=\mathrm{L}_{\sigma}\left(\tilde{g}_{\sigma}^{\infty}\right), \tilde{g}_{u}^{\infty(1)} / \tilde{g}_{u}^{\infty(2)}=\tilde{g}_{\sigma}^{\infty(1)} / \tilde{g}_{\sigma}^{\infty(2)}$ and then $S_{u}^{a}=S_{\sigma}^{b}$. As $\left.\left(\tilde{g}_{t}^{u, n}\right)^{(2)}=-\left(\tilde{g}_{u+1}^{T, n}\right)\right)^{(2)}-\delta_{T}^{n(2)} \rightarrow \tilde{g}_{u}^{\infty(2)}<0$. We may assume that $\tilde{g}_{u}^{n(2)}<0$ and $\left(\tilde{g}_{t}^{u, n}\right)^{(2)}<0$. Let us define $\beta^{n}:=\left(g_{t}^{u, n}\right)^{(2)} / \tilde{g}_{u}^{\infty(2)}$ in $\left.L^{0}\left((0, \infty), \mathcal{F}_{u}\right)\right)$. With $g_{t}^{t-1}=0$ and $\check{g}_{u}^{n}=g_{u}^{n}-\beta^{n} \tilde{g}_{u}^{\infty}$, we rewrite $\delta_{T}^{n}$ as

$$
\delta_{T}^{n}=-g_{t}^{u-1}-\check{g}_{u}^{n}-\beta^{n} \tilde{g}_{u}^{\infty}-g_{u+1}^{T, n}
$$

We may verify that $\check{g}_{u}^{n}=\left(g_{t}^{u-1}\right)^{(2)}\left(S_{u}^{a},-1\right)$ is constant, and so satisfies $\liminf _{n}\left|\check{g}_{u}^{n}\right|<+\infty$. On the set $\Theta_{u-1}^{1}:=\left\{\left(g_{t}^{u-1}\right)^{(2)} \geq 0\right\}$, we have $\mathrm{L}_{u}\left(\check{g}_{u}^{n}\right)=0$. This implies that $\check{g}_{u}^{n} 1_{\Theta_{u-1}^{1}} \in \partial \mathrm{G}_{u}$. On the set $\Theta_{u-1}^{2}:=\left\{\left(g_{t}^{u-1}\right)^{(2)}<0\right\}$, we have $\mathrm{L}_{\sigma}\left(\check{g}_{u}^{n}\right)=0$ hence $\check{g}_{u}^{n} 1_{\Theta_{u-1}^{2}} \in \partial \mathrm{G}_{\sigma}$.

Let us introduce $\bar{g}_{k}:=1_{\sigma=k} 1_{\Theta_{u-1}^{2}} \check{g}_{u}^{n} \in L^{0}\left(\partial \mathrm{G}_{k}, \mathcal{F}_{k}\right)$. Then, $\check{g}_{u}^{n} 1_{\Theta_{u-1}^{2}}=\bar{g}_{u+1}^{T}$. At last, since $\left(-\beta^{n} \tilde{g}_{u}^{\infty}-g_{u+1}^{T, n}\right)^{(2)}=\delta_{T}^{n(2)} \rightarrow 0$ and $u \geq t+1$, the induction hypothesis implies that $-\beta^{n} \tilde{g}_{u}^{\infty}-g_{u+1}^{T, n}=-\breve{g}_{u}^{T, n}+\tilde{\epsilon}_{T}^{n}$ with $\breve{g}_{r}^{n} \in L^{0}\left(\mathrm{G}_{r}, \mathcal{F}_{r}\right)$, $r \geq u$, such that $\sup _{r \geq u} \liminf _{n}\left|\breve{g}_{r}^{n}\right|<\infty$ and $\tilde{\epsilon}_{T}^{n} \rightarrow 0$ a.s. Finally, we write

$$
\delta_{T}^{n} 1_{\Lambda_{t}} 1_{\Gamma_{u}}=-g_{t}^{u-1} 1_{\Gamma_{u}}-\hat{g}_{u}^{T, n} 1_{\Gamma_{u}}+\tilde{\epsilon}_{T}^{n}
$$

where $\hat{g}_{u}^{n}=\check{g}_{u}^{n} 1_{\Theta_{u-1}^{1}} 1_{\Gamma_{u}}+\breve{g}_{u}^{n} 1_{\Gamma_{u}}$ and $\hat{g}_{k}^{n}=\left(\bar{g}_{k} 1_{\Theta_{u-1}^{2}}+\breve{g}_{k}^{n}\right) 1_{\Gamma_{u}}$ if $k \geq u+1$. By construction $\liminf \inf _{n}\left|\hat{g}_{k}^{n}\right|<\infty$ a.s. and $\hat{g}_{k}^{n} \in L^{0}\left(\mathrm{G}_{k}, \mathcal{F}_{k}\right)$ for all $k$.

On $\Lambda_{u}$, we may suppose that $g_{u}^{n} \rightarrow g_{u} \in L^{0}\left(\mathrm{G}_{u}, \mathcal{F}_{u}\right)$ by [13, Lem. 2.1.2] and finally assume w.l.o.g. that $\delta_{T}^{n} 1_{\Lambda_{u}}=-g_{t}^{u} 1_{\Lambda_{u}}-g_{u+1}^{n, T} 1_{\Lambda_{u}}$. We deduce that $\delta_{T}^{n} 1_{\Lambda_{t}}=\delta_{T}^{n} 1_{\Lambda_{t}} 1_{\Lambda_{u}}+\delta_{T}^{n} 1_{\Lambda_{t}} 1_{\Gamma_{u}}$ may be written as $\delta_{T}^{n} 1_{\Lambda_{t}}=-g_{t}^{u-1} 1_{\Lambda_{t}}-g_{u}^{T, n}$ where $\lim \inf \left|g_{u}^{n}\right|<\infty$ a.s. We then reiterate the procedure (i) on $\Lambda_{t}$ where $u$ is necessarily replaced by $\hat{u}>u$. We then conclude on $\Lambda_{t}$ as the number of dates is finite .

(ii) On the set $\Gamma_{t}:=\left\{\liminf _{n}\left|g_{t}^{n}\right|=+\infty\right\}$, we have $\bar{\delta}_{T}^{n}=-\bar{g}_{t}^{T, n}$ where $\bar{\gamma}_{T}^{n}:=\gamma_{T}^{n} /\left|g_{t}^{n}\right|$ and $\bar{g}_{r}^{n}:=g_{r}^{n} /\left|g_{t}^{n}\right|$ for all $r \geq t$. Since $\left|\bar{g}_{t}^{n}\right|=1$, we deduce an 
equality of the type $\bar{g}_{t}^{T, \infty}=0$. As $\bar{g}_{t}^{\infty} \neq 0$, let us consider the first instant $\bar{\tau} \geq t+1$ such that $\bar{g}_{t}^{\tau, \infty}=0$. Then, for any $\operatorname{CPS}\left(Z_{r}\right)_{r \geq t}, Z_{t}, \cdots, Z_{\tau} \in \mathbb{R}_{+} Z_{t}$ by Lemma 4.1. It follows that $Z_{t+1} \in \mathrm{G}_{t}^{*} \cap \mathrm{G}_{t+1}^{*}$ and $\left(Z_{r}\right)_{r \geq t+1}$ is a CPS for the market model from $t+1$ to $T$ defined by $\tilde{\mathrm{G}}_{t+1}=\mathrm{G}_{t} \cup \mathrm{G}_{t+1} \subseteq\left(Z_{t}\right)^{*}$ and $\tilde{\mathrm{G}}_{u}=\mathrm{G}_{u}$ for $u \geq t+2$. Then, the model $\left(\tilde{\mathrm{G}}_{r}\right)_{r \geq t+1}$ satisfies $\mathrm{NA}^{w}$ and $\mathbf{E}$ since $S_{t}^{b} \vee S_{t+1}^{b}$ and $S_{t}^{a} \wedge S_{t+1}^{a}$ are the bid and ask prices at time $t+1$.

Since $g_{t}^{n}+g_{t+1}^{n} \in L^{0}\left(\tilde{\mathrm{G}}_{t+1}, \mathcal{F}_{t+1}\right)$, we may apply the induction hypothesis and deduce that $-\delta_{T}^{n}=\hat{g}_{t+1}^{T, n}$ where $\hat{g}_{u}^{n} \in L^{0}\left(\mathrm{G}_{u}, \mathcal{F}_{u}\right)$ satisfies $\liminf \operatorname{in}_{n}\left|\hat{g}_{u}^{n}\right|<\infty$ a.s. for $u \geq t+2$ and $\hat{g}_{t+1}^{n}=\left(g_{t}^{n}+g_{t+1}^{n}\right) 1_{\liminf _{n}\left\|g_{t}^{n}+g_{t+1}^{n}\right\|<\infty}$. So, assume that $-\delta_{T}^{n}=\left(g_{t}^{n}+g_{t+1}^{n}\right) 1_{\liminf _{n}\left\|g_{t}^{n}+g_{t+1}^{n}\right\|<\infty}+g_{t+2}^{T, n}$ where each $g_{u}^{n}, u \geq t+2$, converges. As liminf $\operatorname{in}_{n}\left|g_{t}^{n}\right|=+\infty$, we deduce by the normalisation procedure an equality $\left(\tilde{g}_{t}+\tilde{g}_{t+1}\right) 1_{\liminf _{n}\left\|g_{t}^{n}+g_{t+1}^{n}\right\|<\infty}=0$ where $\tilde{g}_{t} \in L^{0}\left(\partial \mathrm{G}_{t}, \mathcal{F}_{t}\right)$ and $\left|\tilde{g}_{t}\right|=1$. As $\tilde{g}_{t+1} \in \partial \mathrm{G}_{t+1}$ we deduce that $S_{t}^{a}=S_{t+1}^{b}$ when $\bar{g}_{t}^{\infty(1)}>0$ and $\bar{g}_{t}^{\infty(2)}<0$ and $S_{t}^{b}=S_{t+1}^{a}$ otherwise. On the set $\left\{\bar{g}_{t}^{\infty(1)}>0\right\}$, let us consider the first instant $\hat{\tau} \geq t+1$ such that $\left(S_{t}^{a},-1\right) \in \mathrm{G}_{\hat{\tau}}$. By $\mathbf{E}$ and Remark 2.4, we get that $\hat{\tau} \leq T$ and $\breve{g}_{r}^{n}=g_{t}^{n} 1_{\hat{\tau}=r} \in L^{0}\left(\mathrm{G}_{r}, \mathcal{F}_{r}\right)$ for all $r \geq t+1$. We then write $g_{t}^{n}=\breve{g}_{t+1}^{T, n}$. Similarly, we rewrite $g_{t}^{n}$ on the set $\left\{\bar{g}_{t}^{\infty(1)}<0\right\}$ and we conclude by the induction hypothesis.

\subsection{Proof of Corollary 3.5}

It is trivial that $\Gamma_{\xi} \subseteq\left[\sup _{Q \in \mathcal{M}^{\infty}(P)} \mathrm{E}_{Q} \xi, \infty\right)$. Consider $x \geq \sup _{Q \in \mathcal{M}^{\infty}(P)} \mathrm{E}_{Q} \xi$ and suppose that $x \notin \Gamma_{\xi}$, i.e. $\xi-x \notin \mathcal{L}_{0}^{T}$. As $\mathcal{L}_{0}^{T} \cap L^{1}(\mathcal{P})$ is closed in $L^{1}$ under $\mathrm{NA}^{w}$, we deduce by the Hahn-Banach theorem $\eta \in L^{\infty}$ and $c \in \mathbb{R}$ such that $\mathrm{E}(\eta X)<c<\mathrm{E}(\eta(\xi-x))$ for all $X \in \mathcal{L}_{0}^{T} \cap L^{1}(\mathrm{P})$. Since $\mathcal{L}_{0}^{T}$ is a cone, we deduce that $\mathrm{E}(\eta X) \leq 0$ for all $X \in \mathcal{L}_{0}^{T} \cap L^{1}(\mathrm{P})$. Moreover, as $\mathcal{L}_{0}^{T}$ contains $-L^{0}\left(\mathbb{R}_{+}, \mathcal{F}_{T}\right)$, we deduce that $\eta \geq 0$ and we may suppose $\mathrm{E}(\eta)=1$. Consider $\eta^{\prime}=d Q / d \mathrm{P}$ where $Q \in \mathcal{M}^{\infty}(P) \neq \emptyset$ and choose $\alpha \in(0,1)$ so that $\hat{\eta}:=\alpha \eta+(1-\alpha) \eta^{\prime}$ satisfies $\mathrm{E}(\hat{\eta}(\xi-x))>0$ since $c>0$. By construction, the probability measure $\hat{Q} \sim \mathrm{P}$ such that $d \hat{Q} / d \mathrm{P}=\hat{\eta}$ belongs to $\mathcal{M}^{\infty}(P)$ in contradiction with $\mathrm{E}(\hat{\eta}(\xi-x))>0$, i.e. $x<\mathrm{E}_{\hat{Q}} \xi$.

\subsection{Auxiliary results}

Lemma 4.1. Consider $\tau=\min \left\{u \geq t: g_{t}^{u}=0\right\}$ where $g_{u} \in L^{1}\left(\mathrm{G}_{u}, \mathcal{F}_{u}\right)$, $u \geq t$, and suppose that $\tau \in[t+1, T]$ a.s. Then, for all bounded $C P S Z$, $Z_{r} \in \mathbb{R}_{+} Z_{t}$ a.s. if $r \in[t, \tau]$. 
Proof. Let us define $\hat{g}_{u}=g_{u} 1_{u \leq \tau}$. Since $Z_{T} \hat{g}_{u}^{T}=0$, we deduce that

$$
0=\sum_{u=t}^{T} \mathrm{E}\left(Z_{T} \hat{g}_{u} \mid \mathcal{F}_{t}\right)=\sum_{u=t}^{T} \mathrm{E}\left(Z_{u} \hat{g}_{u} \mid \mathcal{F}_{t}\right) .
$$

Since $Z_{u} g_{u} \geq 0$, we have $Z_{u} \hat{g}_{u}=0$ if $u \geq t$. Since $\{T \leq \tau\} \in \mathcal{F}_{T-1}$ and $\hat{g}_{T} 1_{T \leq \tau}=-g_{t}^{T-1} 1_{\tau=T} \in L^{0}\left(\mathbb{R}^{2}, \mathcal{F}_{T-1}\right)$, we get that $Z_{T} \hat{g}_{T}=0=Z_{T-1} \hat{g}_{T}$ and $Z_{T-1}\left(\hat{g}_{T-1}+\hat{g}_{T}\right)=0$. Suppose that $Z_{u} \hat{g}_{u}^{T}=0$ where $u \geq t+1$. We deduce that $Z_{u-1} \hat{g}_{u}^{T}=0$ since $\hat{g}_{u}^{T}=-g_{t}^{u-1} 1_{u \leq \tau} \in L^{0}\left(\mathbb{R}^{2}, \mathcal{F}_{u-1}\right)$. As $Z_{u-1} \hat{g}_{u-1}=0$ a.s., we get that $Z_{u-1} \hat{g}_{u-1}^{T}=0$, i.e. we may conclude by induction.

In particular, $Z_{u}$ and $Z_{u-1}$ are orthogonal to $\hat{g}_{u, T}$. As $\hat{g}_{u, T} \neq 0$ if $u \leq \tau$, we deduce that $Z_{u} \in \mathbb{R}_{+} Z_{u-1}$ if $u \leq \tau$. The conclusion follows.

Lemma 4.2. Consider $\tau=\min \left\{u \geq t: g_{t}^{u}=0\right\}$ where $g_{u} \in L^{1}\left(\mathrm{G}_{u}, \mathcal{F}_{u}\right)$, $u \geq t$, and suppose that $\tau \in[t+1, T]$ a.s. If $N A^{w}$ holds, there exists a stopping time $\sigma \in[t+1, \tau]$ such that $g_{t} \in \mathbb{R}_{-} g_{\sigma}$ a.s.

Proof. By NA ${ }^{w}$, a bounded CPS $Z$ exists and $Z_{t} \in R_{+} Z_{u}$ a.s. if $u \leq \tau$ by Lemma 4.1. By lemma's proof, $Z_{t} g_{u} 1_{u \leq \tau}=0$ hence $Z_{t} g_{t}=0$. As $g_{t} \neq 0$ a.s., $g_{u} 1_{u \leq \tau}=k_{u} g_{t}$ where $k_{u}=k_{u} 1_{u \leq \tau} \in L^{0}\left(\mathbb{R}, \mathcal{F}_{u}\right)$. Therefore, $\left(1+k_{t+1}^{T}\right) g_{t}=0$ and there is a first instant $\sigma \geq t+1$ such that $k_{u}<0$. We have $g_{\sigma}=g_{\sigma} 1_{\sigma \leq \tau}=k_{\sigma} g_{t}$ so that $g_{t} \in \mathbb{R}_{-} g_{\sigma}$ a.s.

\section{References}

[1] Bouchard B, Kananov Y. and Touzi N. Option pricing by large risk aversion utility under transaction costs. Decisions in Economics and Finance, 2001, 24, 127-136.

[2] Campi L., Schachermayer W. A super-replication theorem in Kabanov's model of transaction Costs. Finance and Stochastics, 2006, 10, 4, 579596.

[3] Cherny A. General arbitrage pricing model. II. Transaction costs, in 'Séminaire de Probabilités XL', 2007, Vol. 1899 of Lecture Notes in Math., Springer, Berlin, 447-461.

[4] De Vallière D., Kabanov Y. and Denis (Lépinette) E. Hedging of American options under transaction costs. Finance and Stochastics, 2009, 13, 1, 105-119.

[5] Dalang E. C., Morton, A. and Willinger, W. Equivalent martingale measures and no-arbitrage in stochastic securities market models. Stochastics and Stochastic Reports, 1990, 29, 185-201. 
[6] Grigoriev P. On low dimensional case in the fundamental asset pricing theorem under transaction costs. Statist. Decisions, 23 (2005), 1, 33-48.

[7] Guasoni P., Lépinette E. and Rásonyi M. The fundamental theorem of asset pricing under transaction costs. Finance and Stochastics, 2012, 16, 4, 741-777.

[8] Guasoni P., Rásonyi M. and Schachermayer, W. The fundamental theorem of asset pricing for continuous processes under small transaction costs. Annals of Finance, 2010, 6, 2, 157-191.

[9] Hodges S., Zhang H. An extended model of effective Bid-Ask spread. http://www.cass.city.ac.uk/__data/assets/pdf_file/0007/ 128068/H. Zhang.pdf

[10] Jouini E., Kallal H. Martingales and arbitrage in securities markets with transaction costs. J. Econ. Theory, 1995, 66, 178-97.

[11] Przemyslaw R. Arbitrage in markets with Bid-Ask spreads. The fundamental theorem of asset pricing in finite discrete time markets with Bid-Ask spreads and a money account. Annal of Finance, 2015, 11,453475.

[12] Schachermayer W. The fundamental theorem of asset pricing under proportional transaction costs in finite discrete time. Mathematical Finance, 2004, 14, 1, 19-48.

[13] Kabanov Y., Safarian M. Markets with transaction costs. Mathematical Theory. Springer-Verlag, 2009.

[14] Kabanov Y., Lépinette E. Consistent price systems and arbitrage opportunities of the second kind in models with transaction costs. Finance and Stochastics, 16, 2011, 1, 135-154.

[15] Lépinette E., Tuan T.Q. General financial market model defined by a liquidation value process. Stochastics, 88 (2016), 3, 437-459.

[16] Rásonyi M. Arbitrage with transaction costs revisited. Optimality and Risk: Modern Trends in Mathematical Finance. Eds. Delbaen F., Rasonyi M., Stricker Ch. Springer, BerlinHeidelbergNew York, 2009. 